\title{
The formation of single-channel and multiple-channel rivers on large slopes
}

\author{
Katarzyna MISIURA ${ }^{1, *}$, Leszek CZECHOWSKI ${ }^{1}$, Piotr WITEK ${ }^{1}$ \\ and Anastasiia BENDIUKOVA ${ }^{1}$
}

1 University of Warsaw, Department of Physics, Institute of Geophysics, Pasteura 7, 02-093 Warszawa, Poland

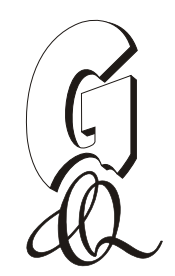

Misiura, K., Czechowski, L., Witek, P., Bendiukova, A., 2016. The formation of single-channel and multiple-channel rivers on large slopes. Geological Quarterly, 60 (4): 981-989, doi: 10.7306/gq.1330

We investigated the formation of different types of rivers depending on slope, total discharge, and grain size. Calculations were performed using numerical package CCHE2D, developed by the National Center for Computational Hydroscience and Engineering. The model is based on the Navier-Stokes equations for depth-integrated two-dimensional turbulent flow and the three-dimensional convection-diffusion equation of sediment transport. For each model we use the same river geometry, suspended load concentration, and bedload transport rate. We distinguish three types of rivers using two methods for classification (single-channel, multiple-channels and transitional). We found that the trend line for transitional rivers is an increasing function of $Q$ in space $(Q, d)$ and that for large $S$ the number of multichannel rivers decreases.

Key words: river classification, multiple-channel rivers, single-channel rivers, sedimentation.

\section{INTRODUCTION}

River systems are important factors that shape the surface of the Earth. The analysis of river systems are based on different criteria. Generally, four pure types of river are considered: single-channel - straight and meandering; multiple-channel braided and anastomosing (e.g., Schumm, 1981; Nichols, 1999). In addition to these "pure" types there exist also "intermediate" (transitional) rivers that have the properties of a few pure types (e.g., wandering rivers - Schumm, 1981).

Lane (1957) and Leopold and Wolman (1957) proposed analysis based on parameters $Q$ and $S$. The space $(S, Q)$ is divided into two regions (meandering rivers, braided rivers) separated by lines specified by simple equations. Another approach is the Rosgen classification (Rosgen and Silvey, 1998; Fig. 1). This classification concerns two basic types: single-channel and multiple-channel rivers. The Rosgen classification is based on the slope and size of the dominant grains of the channel material. Note, however, that for a given slope and grain size there are several possible types of the river according to the Rosgen classification, e.g. A4, F4b, B4, E4b, C4b, and D4b types for the same range of slope $(0.02-0.039)$ and the same grain size

* Corresponding author, e-mail: Katarzyna.Misiura@fuw.edu.pl

Received: May 10, 2016; accepted: October 18, 2016; first published online: December 12, 1016 (gravel). In fact, this classification does not specify the conditions for forming any type of river.

However, according to Leeder (2011: p. 250): "No rigid classification of any single-channel on anything longer than a reach level (a dozen or so channel widths) is universally practicable since many rivers show downstream combinations of sinuosity and braiding. However, many do not and some rational discriminatory classification may be worthwhile". It means that there exists a large group of transitional rivers that are not included in the common classification. Moreover, Leeder (2011: p. 250) stated also: "On the face of it, the magnitude of the energy available to a stream channel and the grain size of the sediment supplied to it might be considered to be primary dynamic variables". Note, the stream energy is given by the formula:

$$
\Omega=\rho g Q S
$$

where: $\rho$-density of liquid, $g$ - gravity, $Q$ - discharge, and $S$-slope.

In the present paper we used a numerical model of the river and tried to determine the values of the parameters - discharge $Q\left(\mathrm{~m}^{3} \mathrm{~s}^{-1}\right)$, slope $S(\mathrm{rad})$ and grain size $d(\mathrm{~mm})$ - necessary for the formation of a given type of river. There are many papers (e.g., Magnuszewski and Gutry-Korycka, 2009a, b) that concentrate on the practical aspects e.g., hydrotechnical, flood control, and protection. However, according to our best knowledge our paper is one of the first presenting classification for this range of parameters (relatively high slope $S$ and small or medium discharge $Q$ ) based on numerical model simulations. 


\section{PARAMETERS OF CONSIDERED MODELS}

The notation of some parameters of the model used in the paper is shown in Table 1 and Appendix $1^{*}$. We used a rather high slope of the channel $S-$ in the range of $0.01-0.04$. We investigated the behaviour of a river with such a range for a few reasons:

- this range is less explored;

- we plan in future to use our calculations for comparison with rivers on Titan, where the typical slope is probably higher (e.g., the range $0.03-0.07$ was found by Perron et al., 2006) than for many terrestrial rivers.

The chosen discharge $Q\left(30-200 \mathrm{~m}^{3} \mathrm{~s}^{-1}\right)$ corresponds with small and medium terrestrial rivers and medium Titanian rivers (e.g., Jaumann et al., 2008; Burr, 2010). The largest Titanian discharge is probably $\sim 1600 \mathrm{~m}^{3} \mathrm{~s}^{-1}$. This value is obtained for an episodic river under the assumption that the flow has short recurrence intervals.

The sizes of grains $d$ of the river bed, bedload, and suspended load are the same for a given model and for each mode we used grains of one size only. The calculations are performed for the following grain sizes: $0.1,1,10,100,200 \mathrm{~mm}$. These sizes are common in terrestrial as well as in Titanian rivers (e.g., Leopold and Emmett, 1976; Tomasko et al., 2005). Taking grains of one size only is a typical physical approach to simplify the situation. In natural rivers it corresponds to cases where one grain-size dominates. The single value (D50) is often used as a parameter characterizing the grain size distribution (e.g., Yalin, 1992: section 1.5).

The size and initial topography of the domain of calculations are given in Figure 2. The length of the domain is $1 \mathrm{~km}$ and its

Ta ble 1

Common parameters used for all models considered

\begin{tabular}{|l|c|}
\hline \multicolumn{1}{|c|}{ Parameter } & Value \\
\hline Gravitational acceleration $\left[\mathrm{m} \mathrm{s}^{-2}\right]$ & 9.817 \\
\hline Specific gravity & 2.65 \\
\hline Kinematic viscosity $\left[\mathrm{m}^{2} \mathrm{~s}^{-1}\right]$ & $1.52 \times 10^{-6}$ \\
\hline Suspended load concentration $\left[\mathrm{kg} \mathrm{m}^{-3}\right]$ & 0.1 \\
\hline Bedload transport rate $\left[\mathrm{kg} \mathrm{m}^{-1} \mathrm{~s}^{-1}\right]$ & 0.1 \\
\hline
\end{tabular}

\section{The Key to the Rosgen Classification of Natural Rivers}

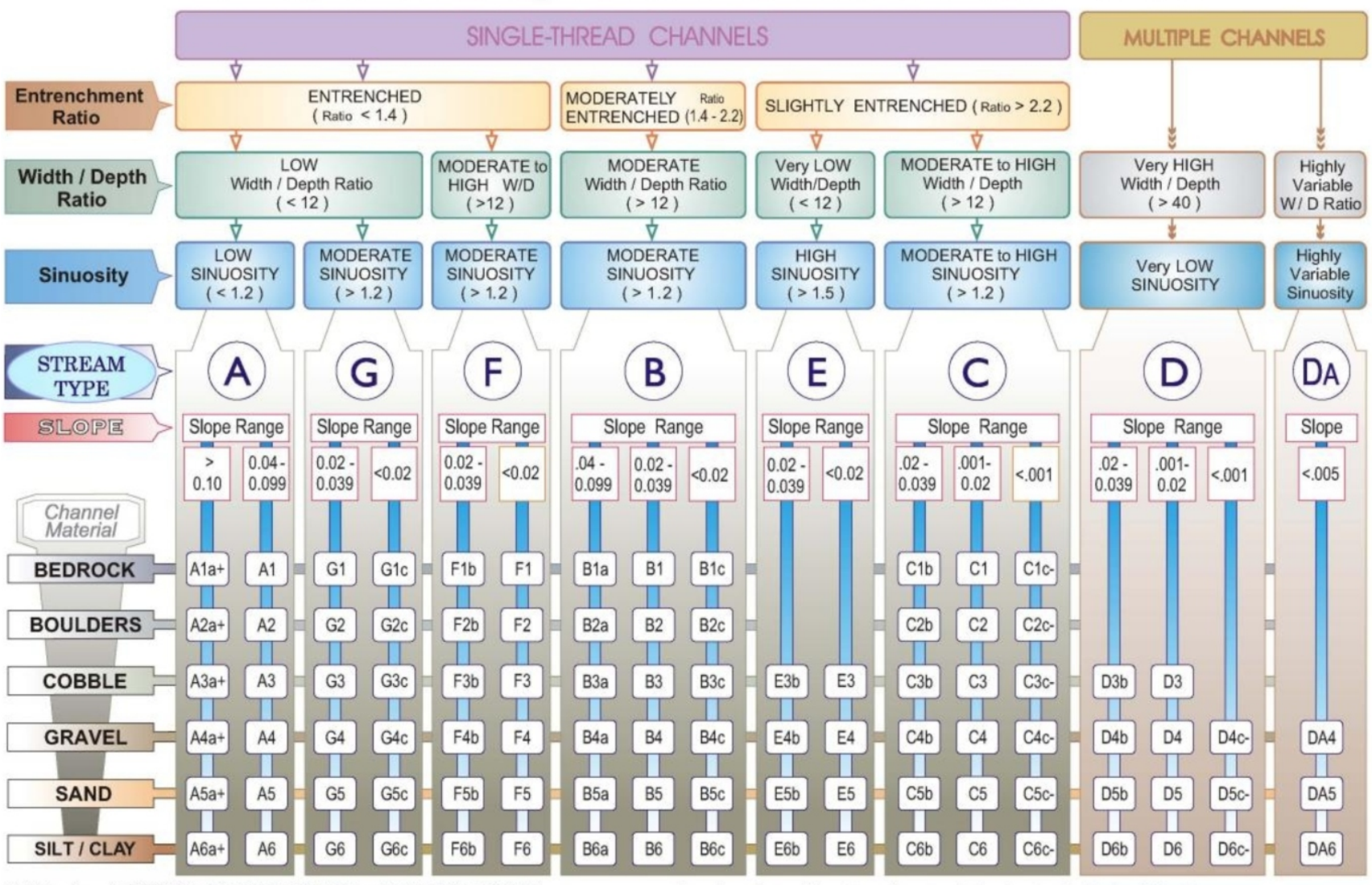

KEY to the ROSGEN CLASSIFICATION of NATURAL RIVERS.

As a function of the "continuum of physical variables" within stream reaches, values of Entrenchment and Sinuosity ratios can vary by $+/-0.2$ units; while values for Width / Depth ratios can vary by $+/-2.0$ units.

(c) Wildland Hydrology 1481 Stevens Lake Road Pagosa Springs, CO $81147 \quad$ (970)731-6100 e-mail: wildlandhydrology@pagosa.net

Fig. 1. Classification of rivers according to Rosgen and Silvey (1998) 
A

Initial Bed Elevation (m)

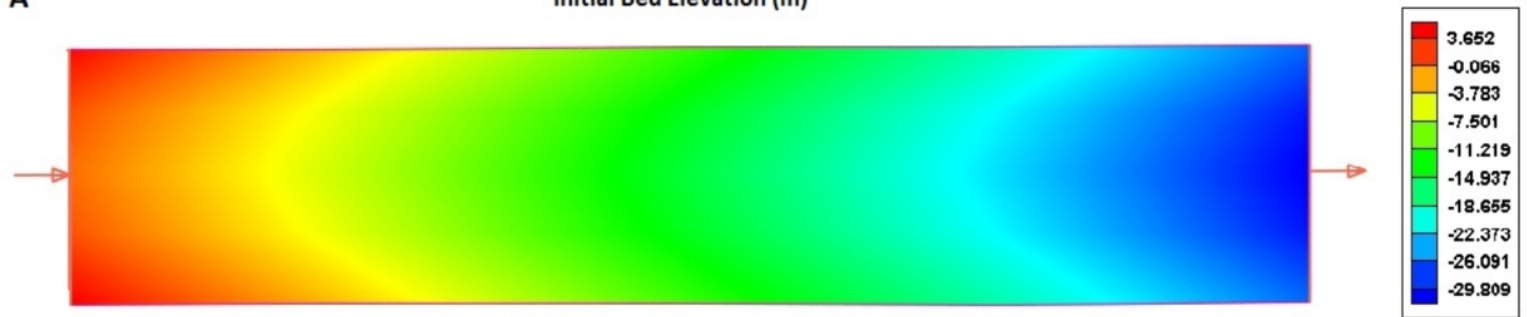

B

Time $=0(\mathrm{~d}): 0(\mathrm{~h}): 0(\mathrm{~m}): 0(\mathrm{~s})$

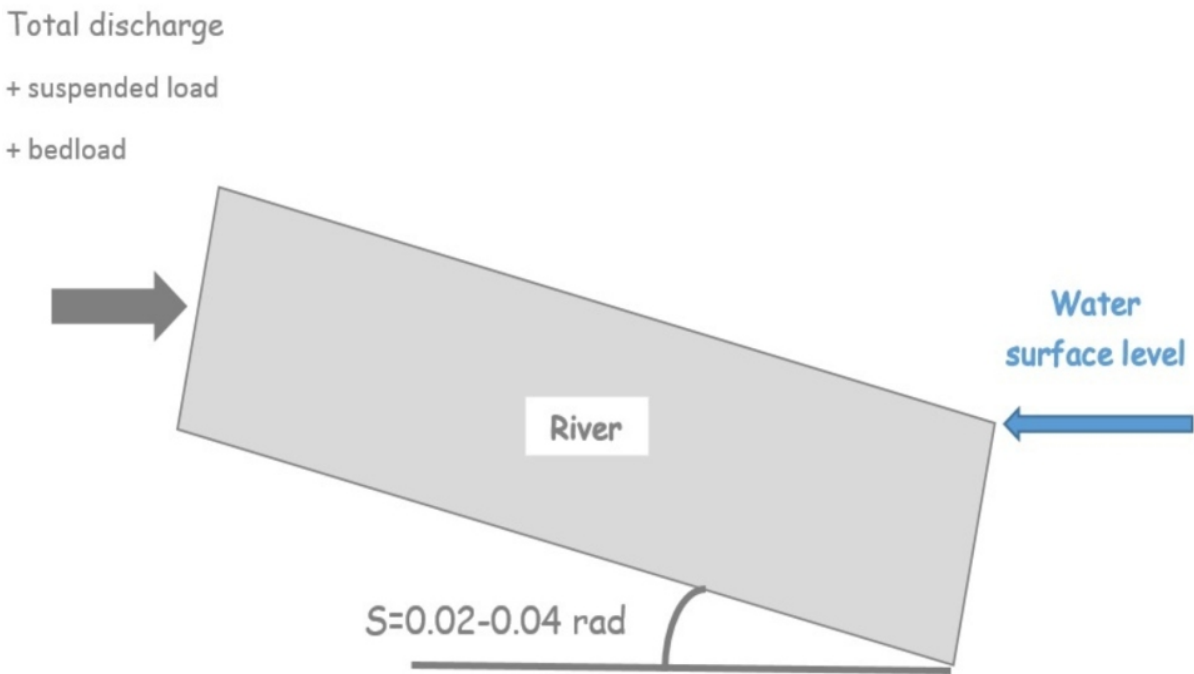

C

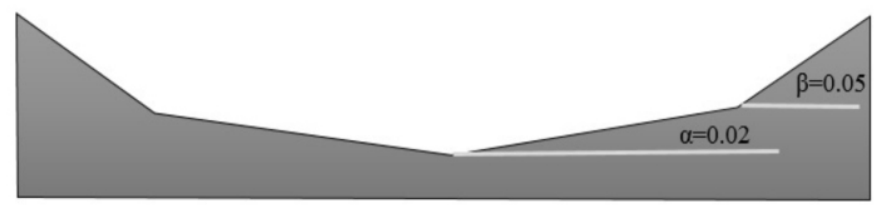

Fig. 2. The initial bed topography and other information about the domain used for our simulations
A - topography, $\mathbf{B}$ - cross section along the river, $\mathbf{C}$ - cross-section across the river; arrows indicate places where initial or boundary conditions are specified

width is $0.2 \mathrm{~km}$. This width allows for the development of a few parallel channels. Note that the domain is constant, so lateral boundaries represent "rigid banks" that cannot be eroded. Fortunately, the modelled river is rather narrow and the stream (or streams) is usually separated from the boundary of the domain by lateral bars. These bars could be subject to erosion, so some lateral motion of the river channel could be also simulated (in the range of the domain).

The initial and boundary conditions for all models are given in Figure 2, Table 1 and Appendix 1. The discharge $Q$, bedload, and suspended load are given at the inflow. For the values of $Q$ used in our simulation, the flow is approximately steady and the bed change is relatively slow. Using the same grain size for the bedload and the suspended load at the inflow is not a crucial assumption because "natural" distributions (i.e. distributions corresponding to current velocity fields and the intensity of turbulence) will be established in a few tens of metres from the begin- ning of the section of the river considered. Then the fine fraction will become the suspended load, while the coarse fraction will become the bedload.

We modelled the evolution of the river for at least 100-200 days. This time is long enough to achieve stabilization (i.e. the initial artificial shape of the water surface changes into a natural one after a few days). Note also that the evolution for the first 11 days could include some transient effects resulting from the artificial initial shape of the channel. Later, transient effects are small and the river could be treated as a natural one.

The Manning coefficient and porosity are given in Figures 3 and 4 . The values of the Manning coefficient for grains up to $1 \mathrm{~mm}$ and for some chosen greater values are taken from Melosh (2011, after Arcement and Schneider, 1989) and Arcement and Schneider (2013). For other larger grains an interpolation is used. A similar procedure is used for the porosity (Fig. 4 based on table 2_1 from McWhorter and Sunada, 1977). 


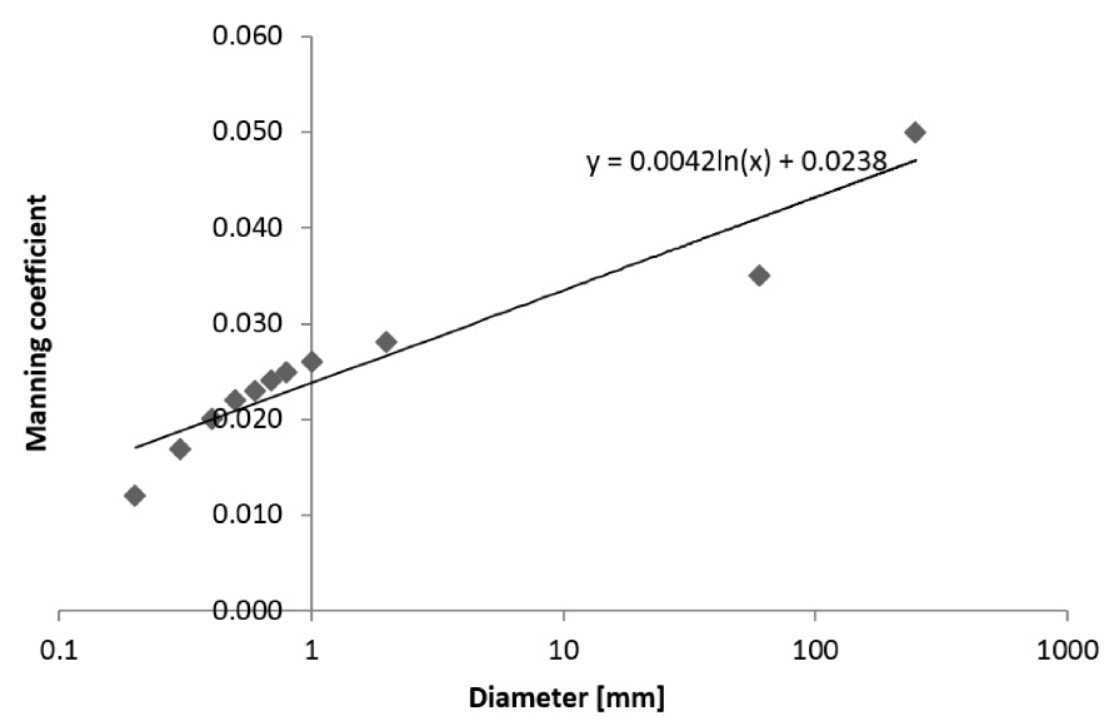

Fig. 3. Manning coefficient versus size of grains (interpolation is made by a logarithm function represented by black, straight line)

Data are obtained by interpolation of the data from Melosh (2011, after Arcement and Schneider, 1989) and Arcement and Schneider (2013)

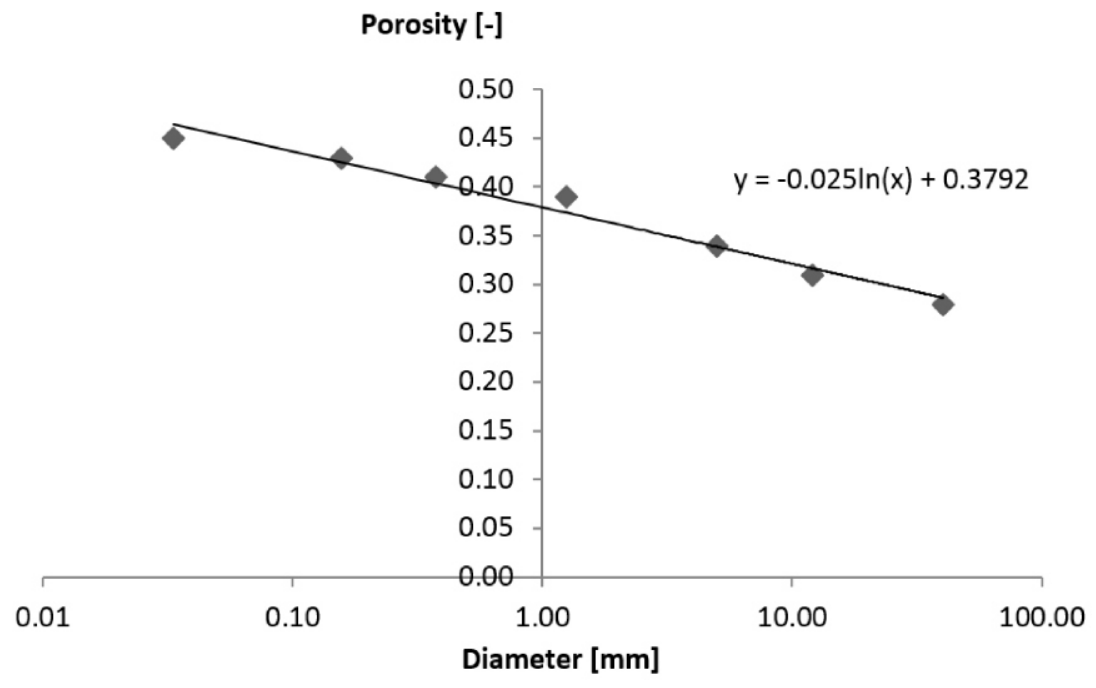

Fig. 4. Porosity versus size of grains

Data are obtained by interpolation of the data from McWhorter and Sunada (1977)

\section{NUMERICAL MODEL}

We chose numerical modelling as our method of investigation. Of course, even the best numerical program uses some simplifications. However, this method has some advantages compared to field research and laboratory models. Contrary to field research, we have good control of the parameters (e.g., discharge). On the other hand, the laboratory models are much smaller than real rivers. We used the same numerical package as in our previous work (Misiura and Czechowski, 2015; Witek and Czechowski, 2015), i.e. CCHE2D package. Basic informa- tion about this program is given below. Full information is in $\mathrm{Wu}$ (2001), Jia and Wang (2001) and Zhang (2006).

In the package CCHE2D, flow dynamics is determined by solution of the Navier-Stokes equations for depth-integrated, two-dimensional turbulent flow and a depth-integrated continuity equation (Jia and Wang, 2001). The transport of sediments is modelled using a three-dimensional convection-diffusion equation. The $\mathrm{k}-\varepsilon$ model of turbulence is used (Zhang, 2006). The package uses the finite element method. The numerical mesh for our model consists of quadrilateral finite elements with 9600 mesh nodes (for details see Zhang, 2006). In these mesh nodes the solver gives: velocity vector, velocity magnitude (i.e. 
the absolute value of the velocity), river depth, bed change (i.e. the difference of the current bed topography from the initial bed topography), bedload, and suspended load. The formulas for bedload and suspended load are given in Wu (2001). The von Karman constant is assumed to be 0.41 (see also discussion in Frenzen and Vogel, 1994). The initial time step is $1 \mathrm{~s}$, however, if necessary it is reduced for stability. The package is widely used for scientific and engineering applications (e.g., for Vistula River; Wu, 2001; Jia and Wang, 2001; Zhang, 2006; Magnuszewski and Gutry-Korycka, 2009a, b).

\section{RESULTS}

The initial shape of the river valley (the computational domain) is very simplified (Fig. 2). The initial morphologic type of river is determined to some degree by this artificial shape, and therefore cannot be used for classification. The channel gradually evolves during the simulation into a "natural" shape. Therefore we used the following procedure for classification of our modelled rivers:

1 - Starting and continuing the simulation for 11 days. The behaviour of the river during this period is not used for the classification of the results.

2 - Continue simulation for the next 100-240 days. During this time some stabilization is achieved indicating that the river could be treated as a natural river.

3 - We used two approaches for river classification: a. based on the spatial and temporary changes; b. based on braid-channel ratio $B$ (see definition of $B$ in Friend and Sinha, 1993).

4 - For the first approach, the behaviour from the 11th day to the end of simulation is used for classification in the following way:

- if the river flows in a single-channel for the whole duration it is classified as a single-channel river;

- if the river flows in a single-channel in some part of the domain but formed a multiple-channel river in another part of domain or during simulation it changes its type (e.g., initially multiple-channel river evolves in a single-channel river) it is classified as transitional type;

- if the river flows in a few channels in the domain it is classified as a multiple-channel river.

5 - For the second approach we used the following procedure:

a. we calculate the coefficient $B$ for the final form of the river (usually after 240 days of simulation);

b. we define three ranges of $B$ :

$-B<1.2-$ single-channel river;

$-1.2 \leq B<1.7$ - transitional river;

$-B \leq 1.7$ - multiple-channel river.

These ranges give good characterization of a river.

The results of the simulations are presented in Figures 5-8. Figure 5 gives some examples of the types of river in our results. Note that most of the examples are "transitional" rivers rather than pure river types.

\section{DISCUSSION}

We found that many of our simulated rivers belong to the transitional type. In fact this indicates that our calculations are realistic because most of the real rivers are also of transitional type (see quotation from Leeder, 2011 in Introduction).
The number of transitional types depends on the chosen number of pure types. Assuming 4 pure types (straight, meandering, braided, anastomosing) then e.g., a slightly sinuous river could be treated as a transitional one between straight and meandering rivers depending on the parameter of sinuosity $K$. In our research we used two pure types: single-channel rivers and multiple-channel rivers. The rivers are classified using parameter $B$ (braid-channel ratio) defined in Friend and Sinha (1993). We use criterion $1.2<B<1.7$ for transitional rivers.

Let us discuss Figure 6. It gives the positions of rivers in space $(Q, d)$ for all considered slopes $S$ (i.e. in fact in $3 D$ space). We used a regression to determine a power function that approximates data for transitional rivers. For all figures we use the same colour code: blue for single-channel rivers, red for transitional rivers, and green for multichannel rivers. The size of the circle corresponds to the value of $S$ (see legend in Fig. 6). We found that the trend line for transitional rivers is an increasing function of $Q$. This is in agreement with the theory (e.g., Leeder, 2011) that gives the formula: $S=a d^{b} Q^{-c}$ where $a, b$, and $c$ are positive numbers. It could be also explained in the following way: the type of river is partly determined by the transport of sediments, so larger d requires higher velocity and consequently larger $Q$. Figure 6 indicates also that single-channel rivers are formed for low $Q$ for all ranges of $d$ and for large $Q$ if $d$ is also large.

The next figure (Fig. 7) presents our results but in 2D space $(d / Q, S)$. As before, for transitional rivers we calculated the trend line represented by a power function. It is also an increasing function. This figure shows better different properties than Figure 6. In particular note that for increasing values of $S$ the number of multichannel rivers is decreasing ( 6 for $S=0.01$ and 2 only for $S=0.04$ ).

A comparison of our results with the results of Lane (1957) and Leopold and Wolman (1957) is given in Figure 8. Note that our results for $S$ are significantly higher than for $S$ in those papers. In fact we do not know of any other systematic research of rivers evolution for our range of $S$. Both our trend line (red line in Fig. 8) and trend lines from Lane (1957) and Leopold and Wolman (1957) are increasing power functions. Note, however, the significantly lower absolute value of the exponent in our function (our 0.026 comparing to 0.23 and 0.44 ).

The criterion used for the choice of transitional rivers in Figure 8 (without $B$ ) is different than the criterion used in Figures 6 and 7 - see description in Results. Generally the results of both criteria are rather similar with some exception, described above in Figure 5. Our lines of trend in Figures 6 and 7 are consistent with formula $S=a d^{b} Q^{-c}$ after Leeder (2011).

\section{CONCLUSIONS}

Using numerical simulations we investigate river evolution for large $S$, i.e. larger than rivers investigated by most of other scientists (e.g., Lane, 1957).

Simulations for small grains (i.e. $d=0.1 \mathrm{~mm}$ ) often meet numerical problems (e.g., too large area of dry regions).

We obtained three main types of rivers (single-channel, multiple-channels, and transitional). There is also a nice example of a braided river (No. 31, $S=0.02$ ).

We used two methods for classification of the rivers (with $B$ calculated for the final form of the river and method without $B$ but considering also changes in time). Both methods are equivalent for most cases.

We found that the trend line for transitional rivers is an increasing function of $Q$ in space $(Q, d)$. This is in agreement with 
Bed change $[\mathrm{m}]$

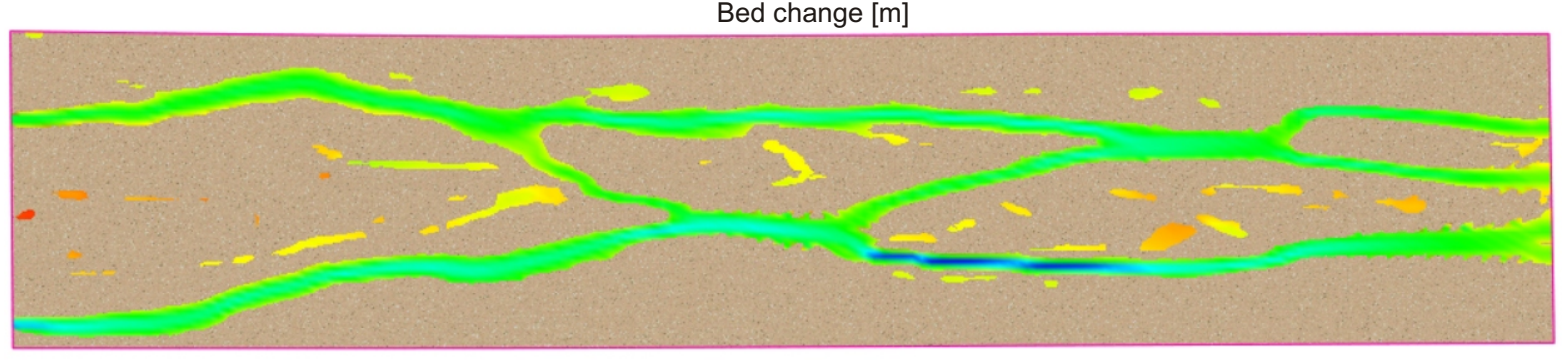

$S=0.02$, No. $31, B=2.31-$ multiple channel river

\section{Bed change $[\mathrm{m}]$}

Time $=240(\mathrm{~d}): \mathbf{6}(\mathrm{h}): \mathbf{1 9}(\mathrm{m}): \mathbf{2 5 . 4 3 0 2 ( \mathrm { s } )}$

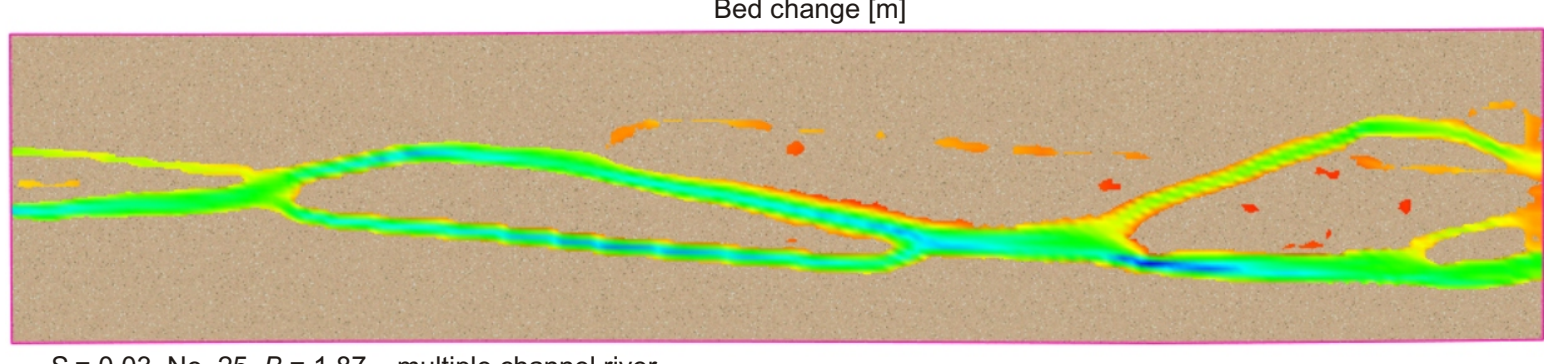

$S=0.03$, No. $25, B=1.87-$ multiple channel river

Time $=240(\mathrm{~d}): 6(\mathrm{~h}): 15(\mathrm{~m}): 8.60462(\mathrm{~s})$

Bed change $[\mathrm{m}]$

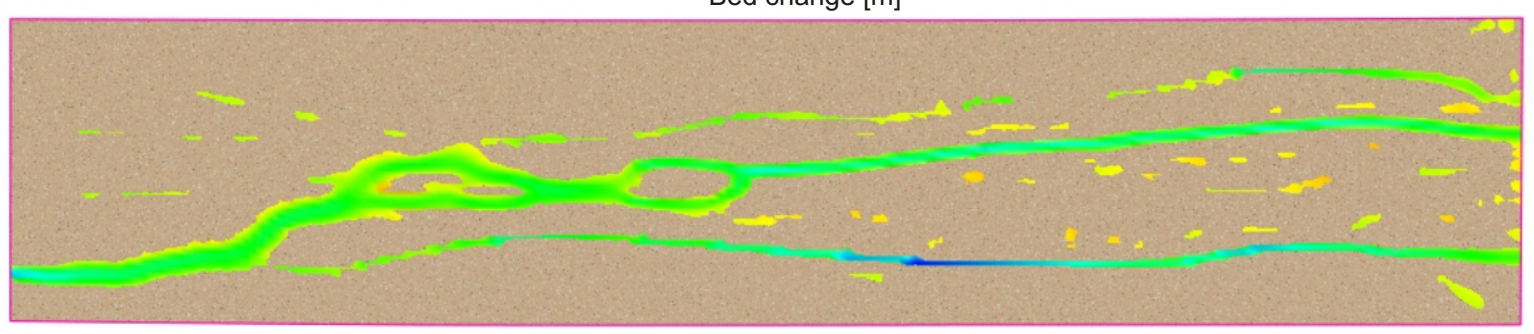

$S=0.04$, No. $21, B=1.2-$ a transitional type. It is a single channel river

in a part of the domain but forms multiple channels in another part of domain $\quad$ Time $=240(\mathrm{~d}): \mathbf{6}(\mathrm{h}): \mathbf{1 3}(\mathrm{m}): \mathbf{3 2 . 1 7 3 4 ( \mathrm { s } )}$

Bed change $[\mathrm{m}]$
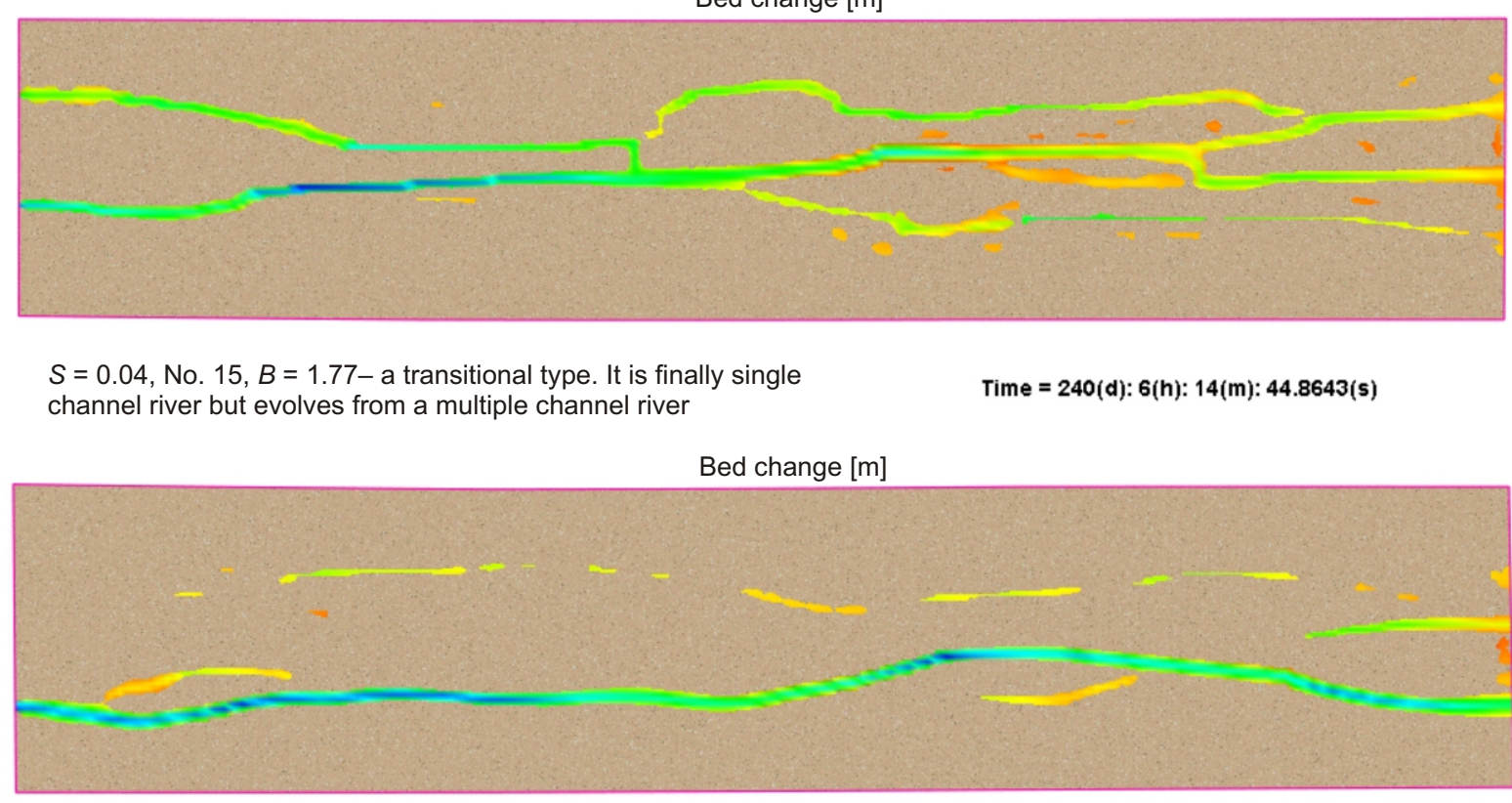

$S=0.02$, No. $15, B=1.03-$ a single channel river

Fig. 5. Some examples of the types of river in our results

Different colour scales are used for different panels; note that $\mathrm{nr} 15$ for $S=0.04$ is classified as transitional river type according to criterion a), but also as a multiple-channel river according to criterion $b$ ) 


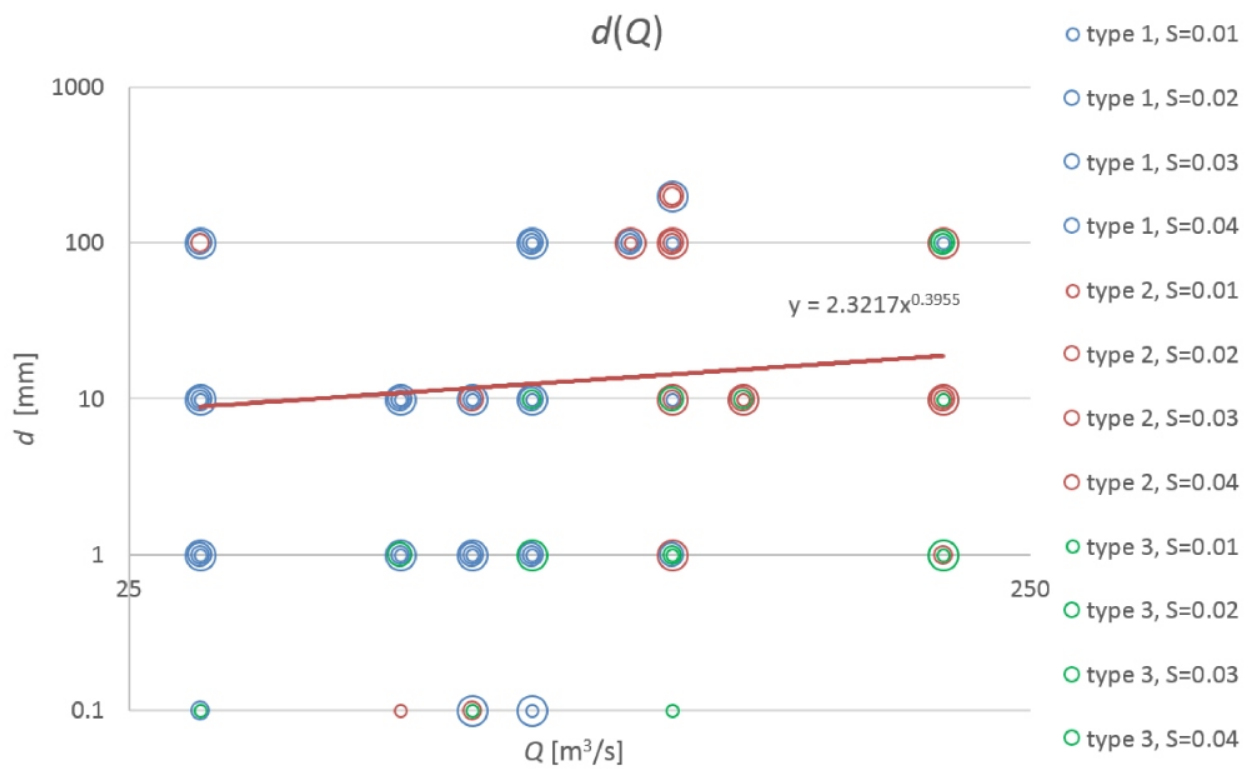

Fig. 6. Positions of obtained rivers in space $(Q, d)$ for all considered slopes $S$ and trend line for transitional rivers

Only circles corresponding to transitional rivers (i.e. red) are used for a regression to determine power function; note that at any given point $(Q, d)$ there could be a few results corresponding to different values of $S$; the power function obtained increases with $Q$ according to prediction of theory (e.g., Leeder, 2011); we divide all rivers based on parameter $B$ (different colours) and on slope (different sizes of circles) see legend next to the graph

\section{$S(d / Q)$}

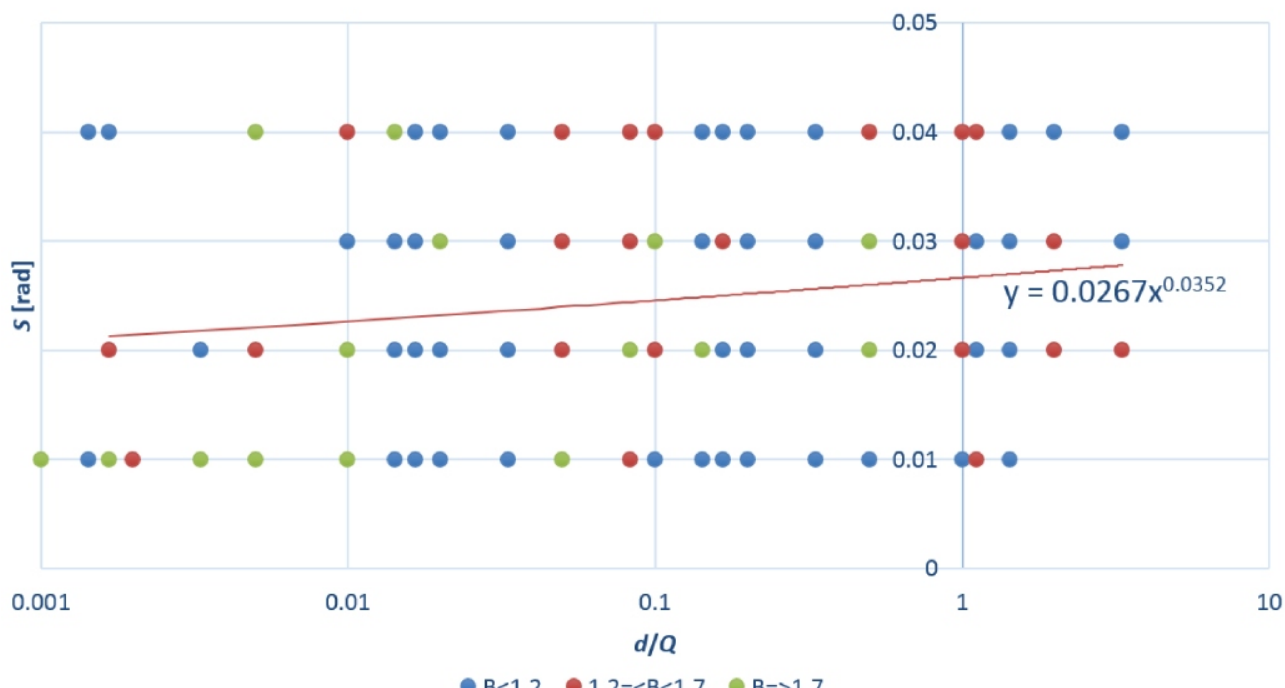

Fig. 7. Positions of obtained rivers in space $(d / Q, S)$ for all considered slopes $S$ and trend line calculated for transitional rivers

Only circles corresponding to transitional rivers (i.e. red) are used for a regression to determine power function; note that the function obtained increases with $Q$ according to prediction of theory

(e.g., Leeder, 2011); we divide all rivers based on parameter $B$ 


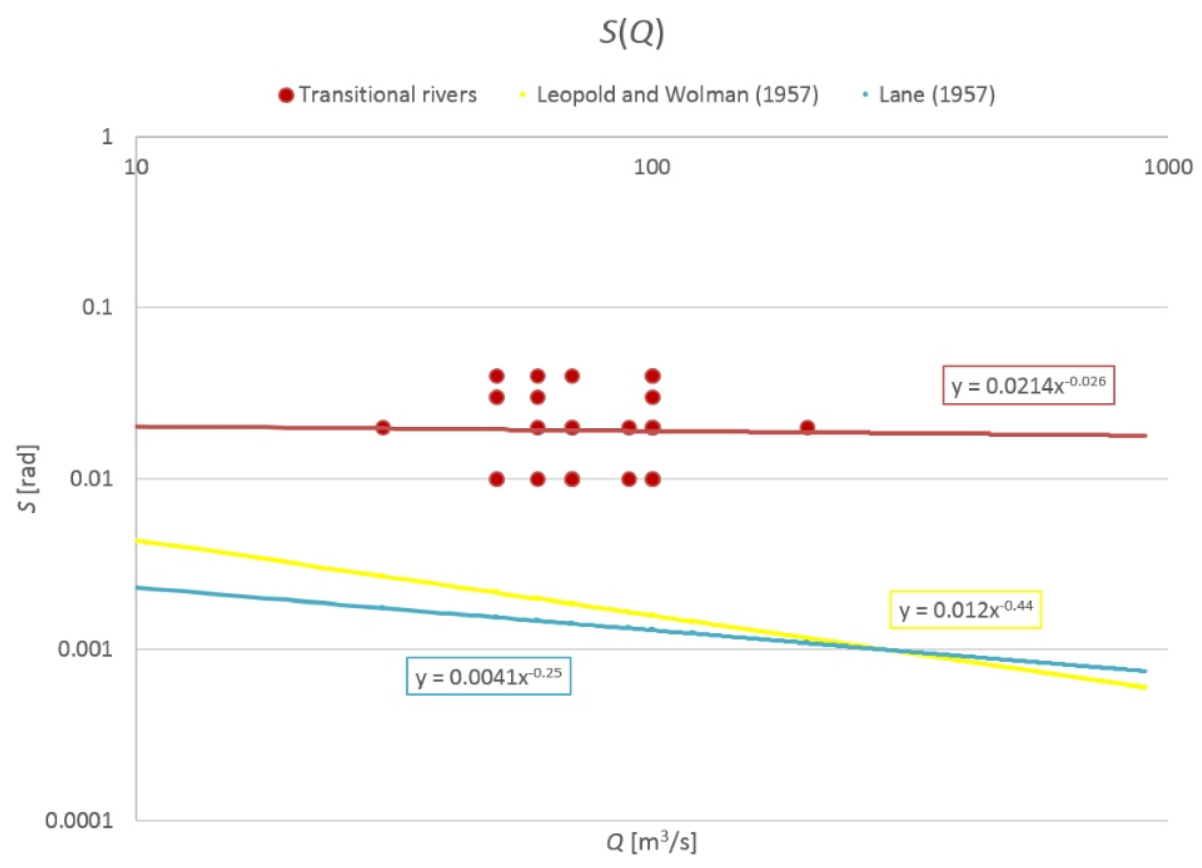

Fig. 8. Positions of simulated rivers in space $(Q, S)$ for all considered $d$ and trend line (red) for transitional rivers (a power function)

The transitional rivers to this graph are chosen according to the criterion described in point 4 in Results (i.e. $B$ is not used); there are shown also braiding and meandering predictors of Lane (1957) and Leopold and Wolman (1957) - the blue and yellow lines

formula: $S=a d^{b} Q^{-c}$. Figure 6 indicates also common single-channel rivers for small $Q$ or for large $Q$ if $d$ is also high.

For large values of $S$ the number of multichannel rivers decreases (Fig. 7).

The exponent in power function for the trend line for large values of $S$ is significantly lower than for low $S$ (Fig. 8).
Acknowledgements. Programs developed by the National Center for Computational Hydroscience and Engineering at The University of Mississippi were used in this research. We are grateful to Prof. A. Wysocka from Faculty of Geology at University of Warsaw. The authors would like to thank the anonymous reviewers for their remarks and valuable comments.

\section{REFERENCES}

Arcement, G., Schneider, V., 1989. Guide for Selecting Manning's Roughness Coefficients for Natural Channels and Flood Plains. United States Geological Survey Water-Supply Paper 2339. http://dpw.lacounty.gov/LACFCD/WDR/files/WG/041615/Guide $\% 20$ for\%20Selecting\%20n-Value.pdf

Arcement, G., Schneider, V., 2013. Guide for Selecting Manning's Roughness Coefficients for Natural Channels and Flood Plains. United States Geological Survey Water-supply. http://www.fhwa.dot.gov/bridge/wsp2339.pdf

Burr, D.M., 2010. Palaeoflood-generating mechanisms on Earth, Mars, and Titan. Global and Planetary Change, 70: 5-13.

Frenzen, P., Vogel, Ch., 1994. On the magnitude and apparent range of variation of the von Karman constant in the atmospheric surface layer. Boundary-Layer Meteorology, 72: 371-392.

Friend, P.F., Sinha, R., 1993. Braiding and meandering parameters. Geological Society Special Publications, 75: 105-111.

Jaumann, R., Brown, R.H., Stephan, K., Barnes, J.W., Soderblom, L.A., Sotin, Ch., Le Mouelic, S., Clark, R.N., Soderblom, J., Buratti, B.J., Wagner, R., Mccord, T.B., Rodirguez, S., Bains, K.H., Cruikshank, D.P., Nicholson, P.D., Griffith,
C.A., Langhans, M., Lorenz, R.D., 2008. Fluvial erosion and post-erosional processes on Titan. Icarus, 197: 526-538.

Jia, Y., Wang, S., 2001. CCHE2D: Two-dimensional Hydrodynamic and Sediment Transport Model for Unsteady Open Channel Flow Over Loose Bed. Technical Report No. NCCHE-TR-2001-1.

Lane, E.W., 1957. A study of the shape of channels formed by natural streams flowing in erodible material. MRD Sediment Series No. 9, U.S. Army Corps of Engineers, Missouri River Div., Omaha, Nebraska.

Leeder, M., 2011. Sedimentology and Sedimentary Basins: from Turbulence to Tectonics. Wiley-Blackwell.

Leopold, L.B., Emmett, W.W., 1976. Bedload measurements, East Fork River, Wyoming. Proceedings, National Academy of Sciences, 73: 1000-1004.

Leopold, L.B., Wolman, M.G., 1957. River channel patterns: braided, meandering and straight. USGS Professional Paper, 282-B.

McWhorter, D.B., Sunada, D.K., 1977. Ground-water hydrology and hydraulics. Water Resources Publication, 290. 
Magnuszewski, A., Gutry-Korycka, M., 2009a. Reconstruction of the Vistula River extreme floods passage through the City of Warsaw in natural river conditions (in Polish with English summary). Prace i Studia Geograficzne, 43: 141-151.

Magnuszewski, A., Gutry-Korycka, M., 2009b. Flood waters flow in the contemporary Vistula River channel (in Polish with English summary). Prace i Studia Geograficzne, 43: 153-162.

Melosh, H.J., 2011. Planetary Surface Processes. Cambridge Planetary Science.

Misiura, K., Czechowski, L., 2015. Numerical modelling of sedimentary structures in rivers on Earth and Titan. Geological Quarterly, 59 (3): 565-580.

Nichols, G., 1999. Sedimentology and Stratigraphy. Wiley-Blackwell, Oxford.

Perron, J.T., Lamb, M.P., Koven, C.D., Fung, I.Y., Yager, E., Adámkovics, M., 2006. Valley formation on Titan. Journal of Geophysical Research, 111: E11001.

Rosgen, D., Silvey, L., 1998. Field Guide for Stream Classification. Wildland Hydrology.

Schumm, S.A., 1981. Evolution and response of the fluvial system sedimentologic implications. SEPM Special Publication, 1: 19-29.
Tomasko, M.G., Archinal, B., Becker, T., Bézard, B., Bushroe, M., Combes, M., Cook, D., Coustenis, A., de Bergh, C., Dafoe, L.E., Doose, L., Douté, S., Eibl, A., Engel, S., Gliem, F., Grieger, B., Holso, K., Howington-Kraus, E., Karkoschka, E., Keller, H.U., Kirk, R., Kramm, R., Küppers, M., Lanagan, P., Lellouch, E., Lemmon, M., Lunine, J., McFarlane, E., Moores, J., Prout, G.M., Rizk, B., Rosiek, M., Rueffer, P., Schröder, S.E., Schmitt, B., See, C., Smith, P., Soderblom, L., Thomas, N., West, R., 2005. Rain, winds and haze during the Huygens probe's descent to Titan's surface. Nature, 438: 765-778.

Witek, P.P., Czechowski, L., 2015. Dynamical modelling of river deltas on Titan and Earth. Planetary and Space Science, 105: 65-79.

Wu, W., 2001. CCHE2D Sediment Transport Model (Version 2.1). Technical Report No. NCCHE-TR-2001-3.

Yalin, M.S., 1992. River Mechanics. Pergamon Press, Oxford.

Zhang, Y., 2006. CCHE-GUI - Graphical Users Interface for NCCHE Model User's Manual - Version 3.0. Technical Report No. NCCHE-TR-2006-02. 